\title{
On the Transformation-Sequence.
}

By R. F. Muirhead, M.A., B.Sc.

This paper is a continuation of my paper, "On a Method of Studying Displacement," in last year's Proceedings. In that paper I showed how the chief theorems as to the displacements of rigid bodies could be simply demonstrated by the use of what I called a Displacement-chain or Displacement-sequence.

In this paper I complete the treatment of displacement accompanied by perversion, by adding an investigation of the threedimensional perversion-sequence; and thereafter I show how the sequence method may be extended to other transformations besides those corresponding to rigid displacements and perversions.

From one point of view we may regard a displacement of a rigid body as a change by which every point in the body is transformed into another point of space, and by imagining the body unlimited in extent, we get the idea of a transformation by which we pass from any one point of space to some point which is in general different from it.

In the same way, starting with two bodies $F$ and $F_{1}$, such that $F_{1}$ is the perverted reproduction of $F$, i.e., such that $F_{1}$ is related to $F$ as the right hand to the left, or as an object to its image in a plane mirror (but not necessarily in the special relative position which object and image occupy)-and supposing each of these bodies indefinitely extended-we get a space-transformation which is analogous to a rigid-displacement-transformation, but differs from it in the peculiar way indicated.

The nature of the perversion-sequence in two dimensions was discussed in the previous paper: let us now study it in three dimensions.

Let $\mathrm{ABCDE}$ (Fig. 15) be a perversion-sequence, with reference to a body $F$ and its perversion $F_{1}$; so that $A$ in $F$ corresponds to $B$ in $F_{1}, B$ in $F$ to $C$ in $F_{1}$, and so on. Join $P, Q, R, S$ the mid-points of $A B, B C, C D, D E$, and join $A C, B D, C E, A E$.

Then $\mathrm{AB}=\mathrm{BC}=\mathrm{CD}=\mathrm{DE} ; \quad \mathrm{AC}=\mathrm{BD}=\mathrm{CE}$. By reasoning similar to that in the first half of p. 125, Vol. $X V$., we can show that the points $E$ and $A$ are on the same side of the plane BCD. 
Hence the dihedral angles $A \overline{B D} C$ and $E \overline{D B C}$ (at the edge $D B$ ) are equal, since they are corresponding angles in the tetrahedrons $A B D C$ and $E D B C$ which are symmetrically equal to one another. Hence the planes $\mathrm{ABD}$ and $\mathrm{EBD}$, making equal angles with the plane CBD on the same side of it, coincide; so that $A B D E$ is a plane trapezium having $\mathrm{AB}=\mathrm{DE}$ and $\mathrm{BD} \|$ to $\mathrm{AE}$.

$\mathrm{QR}$ which is $\|$ to $\mathrm{BD}$, is $\|$ to $\mathrm{AE}$

Also $P Q$ is || to $A C$ and $R S$ to $C E$.

Thus PQ, QR, RS being parallel to three lines in the plane ACE, and conterminous, are coplanar.

Similarly Q, R, S, T are coplanar, . PQRST are so. By induction we see that the mid-points of the successive steps $\mathrm{AB}, \mathrm{BC}$... in the perversion-sequence all lie in the same plane. This plane, then, is unaltered in position by the perversion, though its points are not unaltered.

Again, since $A$ in $F$ corresponds to $B$ in $F_{1}$ the plane PQRS ... will appear in $F$ as seen from $A$, as the perverse of the corresponding plane in $F_{1}$ as seen from $B$. But $A$ and $B$ are on opposite sides of that plane. Hence the points of the plane PQRS .. . before and after transformation, are congruent when looked at from the same side of the plane.

Hence, as shown in Vol. XV., p. 120, there will be one point that will remain unaltered by the transformation in question.

Thus the most general perverted displacement in three dimensions may be analysed into a reflection with reference to a plane PQRS . . . , together with a rotation about a line perpendicular to that plane passing through the centre of the regular polygon PQRST ...

The amount of the rotation is equal to the angle between $P Q$ and $\mathrm{QR}$, which is half that between $\mathrm{AC}$ and $\mathrm{CE}$.

If we extended this method of analysis to perverted displacement in $n$ dimensions we should find that in general a certain flat space of $n-1$ dimensions in the body of reference is unaltered in position but transformed so as to become congruent with itself, so that such a perverted displacement would be equivalent to a reflection in that $(n-1)$-flat together with a displacement of the ordinary sort by which the points of the $(n-1)$-fiat would move in their most general manner consistent with the maintenance of that $(n-1)$-flat in its original position. 
Let us now generalize by one degree, the space transformation associated with a displacement (with or without perversion), by supposing the body $F$ to be not merely displaced, but also changed in magnitude, its shape however remaining unchanged. This amounts to the condition that the line joining each pair of points of $F$ is altered in the same ratio, or that straight lines remain straight lines, and angles are unaltered in magnitude.

This we may call a Conformal Transformation. Let then ABCDE . . (Fig. 16) be a Conformal Transformation-sequence, and first let us suppose it without perversion, and confined to twodimensional space.

Since $\mathrm{ABC}$ transforms in to $\mathrm{BCD}$, the triangles are similar, and in fact it is obvious that $\triangle B C D E \ldots$ forms an equiangular polygon, whose sides are in continued proportion. The eye sees at once that by producing the sequence indefinitely, ${ }^{*}$ we should get indefinitely near to a point $O$ which would be unaltered by the transformation, and which would be the pole of an equiangular spiral circumscribed about the polygon.

But the point $O$ may be got more directly by describing on $A B$ as chord, a circle which will touch $B C$ at $B$, and lie on the same side of $\mathrm{BC}$ as $\mathrm{AB}$ does; and another circle on $\mathrm{BC}$ as chord, touching $\mathrm{AB}$ on the side on which $\mathrm{C}$ lies. These circles will cut again in a point $O$, which will correspond to itself, since the triangles $\mathrm{OAB}, \mathrm{OBC}$ are obviously similar.

Thus in Conformal Transformation, there is one point $O$ which is unaltered. This is what is called the Centre of Similitude of $F$ and $F^{\prime}$ in the theory of two bodies directly similar in one plane. If we join $O$ to $A, B, C, D \ldots$ and cut off from $O B, O C, O D \ldots$ lengths $\mathrm{O} a, \mathrm{O} b, \mathrm{O} c \ldots$ equal respectively to $\mathrm{OA}, \mathrm{OB}, \mathrm{OC} \ldots$ we see that the transformation in question may be analysed into a rotation about $O$ through an angle equal to $A O B$, (bringing $A B C$... to $a b c \ldots$ ) together with a radial contraction or expansion by which each radius from $O$ is changed in the same ratio (bringing $a b c \ldots$ to $\mathrm{BCD}$...).

Note that three points $\mathrm{A}, \mathrm{B}, \mathrm{C}$ of a sequence are sufficient to

* This is true if the ratio $\mathrm{AB}: \mathrm{BC}$ is greater than unity ; on the contrary supposition we should have to trace the sequence backwards to get towards 0 . This note will apply to later passages : but the application may be left to the reader. 
determine the transformation completely. Suppose, however, two similar triangles $L M N$ and $L^{\prime} M^{\prime} N^{\prime}$ are given, and it is required to find their centre of similarity. Join $\mathrm{LL}^{\prime}, \mathrm{ML}^{\prime}$, and on $\mathrm{L}^{\prime} \mathrm{M}^{\prime}$ describe a $\triangle L^{\prime} M^{\prime} L^{\prime \prime}$ directly similar to the $\triangle L^{\prime} M L^{\prime}$. Obviously $L L^{\prime} L^{\prime \prime}$ form a sequence, and the preceding construction for $O$ can therefore be applied.

Next suppose $F^{\prime}$ to be perversely similar to $F$. The corresponding space-transformation may be called a perversely-conformal transformation.

The transformation-sequence in this case (see Fig. 17) is clearly a zig-zag equiangular figure with sides in continued proportion, and parallel alternately, and the construction evidently tends towards a certain point $O$ which would be unaltered by the transformation.

Let us divide $A B, B C, C D \ldots$ in $P, Q, R \ldots$ in the same ratio as $\mathrm{AB}: \mathrm{BC}$ or $\mathrm{BC}: \mathrm{CD}$.

Then $\quad A P: A B:: B Q: B C \quad \therefore A P: B Q:: A B: B C$

Hence $\quad \mathrm{PB}=\mathrm{BQ} \quad \therefore \angle \mathrm{BPQ}=\angle \mathrm{BQP}$.

From this it follows easily that $\angle \mathrm{BQP}=\angle \mathrm{CQR}$ and that $P, Q, R$ are collinear, and lie in a straight line which passes through $\mathrm{O}$, the intersection of $\mathrm{AC}$ and $\mathrm{BD}$.

PQR ... forms a sequence of collinear points whose distances from $O$ are changed by the transformation in a fixed ratio, while the position of the line as such is unaltered.

It is obvious that the interior bisectors of such angles as ABC, BCD are all parallel, and unaltered in direction by the transformation.

If $\mathrm{YOY}^{\prime}$ be drawn through $\mathrm{O}$ perpendicular to PQR...O, we see that the transformation is equivalent to a reflection in the line $O P$, together with a linear contraction or expansion to or from $O P$, and another linear contraction or expansion to or from $O Y$; or, more briefly, to a reflection in $O P$ and a radial contraction or expansion to or from the centre $O$, by which each radius is changed in the same ratio.

Consider now the direct Conformal Transformation in three dimensions, by which a body $F$ is transformed into a body $F^{\prime}$ similar to $F$ and similarly situated on its base.

The Transformation-sequence ABCDE ... in this case is a skew 
equiangular polygon whose sides are in continued proportion, and whose successive planes $\mathrm{ABC}, \mathrm{BCD}, \mathrm{CDE}$, etc., make equal angles in pairs, but so that $A$ and $E$ are on opposite sides of the plane BCD.

In fact it is a kind of skew rectilinear spiral, and obviously leads towards a pole which will be unaltered by the transformation.

By applying to it the construction and reasoning of $p$. 122, Vol. XV., it appears that as in the case of the Displacement-sequence the directions of the shortest distances between the interior bisectors of successive angles of the sequence, are unaltered, but they are no longer conterminous, nor in the same straight line. We see, however, that they are all in the same direction. All lines of $F$ in this direction, and all planes perpendicular to this direction in $F$ are unaltered in direction by the transformation.

It follows that if we take a sequence of such planes, the distance between any two in sequence will diminish in geometric progression as we go forwards (or backwards). Hence we shall approach a plane which is unaltered in position as well as in direction. When three of the planes in sequence are given we can get this unique plane by an obvious construction.

The points of this unique plane are in general changed by the transformation, but as it does not move out of itself, it can be made self-coincident in all its points by the direct conformal transformation in two dimensions.

We now see that the three-dimensional direct conformal transformation may be analysed into an expansion or contraction in a certain direction, leaving a certain plane fixed, together with a two-dimensional conformal transformation. Or we may state it as a rotation about a certain line which meets the unique plane in a fixed point, together with a radial expansion or contraction about that point, such that all radii are changed in the same ratio.

Thus one point, one line, and one plane retain their positions after transformation.

Take next, the Perverse Conformal Transformation. Let $A B C D E \ldots$ be a sequence, and $P, Q, R, S \ldots$ the points which divide $A B, B C, \ldots$ in the same ratio as $A B$ to $B C$, etc.

$A s$ in the corresponding construction in two dimensions, it follows that $P B=B Q, Q C=C R$, etc., and that $\angle B Q P=\angle C Q R$.

Hence the plane $P Q R$ makes equal angles with the planes 
$B P Q$ and $C Q R$, and therefore the dihedral angles $\overline{P Q R} B$ and $\mathbf{R} \overline{\mathrm{PQ}} \mathrm{B}$ are supplementary.

But the tetrahedron CQRS is perversely similar to the tetrahedron BPQR. Hence the dihedral angles $\overline{C Q R S}$ and $B \overline{P Q} R$ are equal. Therefore the dihedrals $\mathrm{C} \overline{\mathrm{QRS}}$ and $\mathrm{P} \overline{\mathrm{QR}} \mathrm{S}$ are supplementary. Hence the planes $\mathrm{PQR}$ and QRS coincide.

Thus the points PQRS... form a coplanar sequence, and as in the corresponding case of perverted displacement, the plane containing them is unaltered by the transformation, and has its points directly conformal to one another.

We see, in fact, that the general Perversely-Conformal Transformation may be analysed into a reflection in the plane $P Q R$... together with a rotation about an axis perpendicular to this plane, and through the pole of the rectilinear spiral $P Q R \ldots$, and an axial contraction towards or expansion from that pole as fixed centre.

Thus there is a point which remains unaltered, a line passing through it which retains its position, and a plane through the point and perpendicular to the line which also retains its position.

Next above the Conformal Transformation in generality, and including it as particular case, comes the Linear Transformation which distinguishes itself by the property that every straight line of $F$ remains a straight line in $F^{\prime}$, while corresponding points are the intersections of corresponding lines.

In such a corresponding transformation parallel or concurrent lines would remain parallel or concurrent, hence an harmonic ratios of pencils and ranges would remain unaltered, and therefore the ratios between segments of the same line would remain unaltered.

For the general Linear Transformation in two dimensions, four points of a sequence would be necessary and sufficient for its determination, and the production of the sequence $A, B, C, D$ might be thus carried out :

Join AC, BD and let them cut in $L$.

Divide $B D$ in $M$ so that $B M: M D:: A L: L C$.

Join $C M$ and produce to $E$ so that $C M: M E:: B L: L D$.

Then $\mathbf{E}$ is the next point of the sequence.

A similar construction would hold in three dimensions, five points of the sequence being given, the modification required for a perverse transformation being obvious in either case. 
The analysis by the aid of the Transformation-sequence would clearly lead to somewhat similar results in the case of the general Linear Transformation, as we have worked out for the Conformal case. The expansions and contractions would, however, no longer be equal in all directions, but decomposible into linear expansions or contractions in mutually perpendicular directions.

These transformations are, of course, from another point of view, simply transformation of coordinates, which may be taken as areals in the case of two dimensions, and tetrahedral coordinates in the case of three dimensions.

The general case would correspond to the case where any triangle (or tetrahedron) is taken as the new triangle of reference; the Conformal Transformation would correspond to the case where the new triangle (or tetrahedron) is similar to the old one; and Displacement to the case when the new triangle (or tetrahedron) of reference is congruent to the old one.

I may mention that, through the kindness of Professor Lamb, of Owens College, I recently learnt that the idea of the Displacement-sequence, and also the construction for displacement in two and three dimensions which I gave in my paper in Vol. XV., are to be found in a paper by Professor Crofton, pp. 25-27 of Vol. 5 of the Proceedings of the London Mathematical Society.

It is interesting to remark that cardboard models of the Conformal Transformation-sequence in three dimensions can be easily constructed as follows :

Take a triangular piece of cardboard $\mathrm{ABO}$, of any shape (see Fig. 18) and draw lines BC, CD, DE, EF ... alternately parallel to one another.

Let half the thickness of the cardboard be cut through along the lines $\mathrm{BC}, \mathrm{CD}, \mathrm{DE}$, either (i.) all on the same side of the cardboard, or (ii.) alternately on opposite sides. The cardboard can now be bent about these edges, so as to make equal angles between successive planes ABC, BCD, CDE, etc. Case (i.) gives the model of a perverse transformation-sequence, and case (ii). that of a direct one.

To get the particular case of the displacement-sequence, it is only necessary to make $\mathrm{BD}$ and $\mathrm{AC}$ parallel, or $\mathrm{O}$ infinitely distant. 